\title{
Tunneling magnetoresistance in devices based on epitaxial NiMnSb with uniaxial anisotropy
}

\author{
J. Liu*, E. Girgis, P. Bach, C. Rüster, C. Gould, G. Schmidt, and L.W. Molenkamp \\ Physikalisches Institut der Universität Würzburg, \\ Am Hubland, 97074 Würzburg, Germany and \\ *Engineering Research Center for Semiconductor Integrated Technology, \\ Institute of Semiconductors, Chinese Academy of Sciences, \\ P.O. Box 912, Beijing 100083, P.R. China
}

(Dated: November 18, 2018)

\begin{abstract}
We demonstrate tunnel magnetoresistance (TMR) junctions based on a tri layer system consisting of an epitaxial NiMnSb, aluminum oxide and CoFe tri layer. The junctions show a tunnelling magnetoresistance of $\Delta R / R$ of $8.7 \%$ at room temperature which increases to $14.7 \%$ at $4.2 \mathrm{~K}$. The layers show clear separate switching and a small ferromagnetic coupling. A uniaxial in plane anisotropy in the NiMnSb layer leads to different switching characteristics depending on the direction in which the magnetic field is applied, an effect which can be used for sensor applications.
\end{abstract}




\section{INTRODUCTION}

Tunneling magnetoresistance (TMR) was first observed in ferromagnet-insulatorferromagnet (FM-I-FM) junctions using ferromagnetic metals (like $\mathrm{CoFe}$, Co or $\mathrm{Fe}$ ) and $\mathrm{Al}_{2} \mathrm{O}_{3}$ tunnel barriers 1, 2]. Thereafter TMR has been intensively studied due to its applications in magnetic random-access-memory (MRAM) and magnetic sensors [3, 4]. The magnitude of the tunneling magnetoresistance depends strongly on the density of states in the electrodes and in most cases can be calculated using Jullieres formula [5] $]$.

$$
T M R=\frac{R_{A P}-R_{P}}{R_{A P}}=\frac{2 P_{1} P_{2}}{1+P_{1} P_{2}}
$$

where $\mathrm{P}_{1}$ and $\mathrm{P}_{2}$ are the spin polarization for the two FMs. From this formula it can immediately be inferred that a higher spin polarization in the electrodes also leads to an increase of the TMR. For a material in which all tunnelling electrons have the same spin direction, the magnetoresistance can theoretically be infinite. This fact has led to an increasing interest in the possibility of using half metallic materials for TMR junctions and indeed experiments with $\mathrm{La}_{0.7} \mathrm{Sr}_{0.3} \mathrm{MnO}_{3}[$ 6] have already yielded extremely high TMR values.

Other materials for which half metallic behaviour is predicted can be found among the so called Heusler alloys [7], which are typically ternary compounds with metallic conductivity. One candidate which has been subject to several theoretical calculations is NiMnSb which is predicted to be $100 \%$ spin polarized even at room temperature [8]. Because of this theoretical prediction the material and its deposition have been studied by various groups and it is now possible to obtain fully epitaxial thin films of NiMnSb 9 , 10]. Positron annihilation experiments on bulk material have already indicated that NiMnSb is indeed half metallic 11 , 12]. Despite the high quality of epitaxial layers, however, no half-metallicity in thin layers has been observed up to now. A possible explanation is that most investigation methods for layers are surface sensitive and surface states may cause the appearence of additional states in the gap of the bandstructure of the NiMnSb, leading to a reduced spin polarization[13].

However, even with a spin polarization of slightly less than $100 \%$ NiMnSb can still be interesting for TMR devices and a number of attempts have been made to introduce single

or polycristalline NiMnSb layers in various kinds of structures 14]. The magnetoresistance obtained up to now, however is comparable to or even smaller than that achieved using traditional metallic ferromagnets. 
We have fabricated a layer stack based on a 2.5 (???) nm thick epitaxial NiMnSb layer grown on a lattice matched semiconductor substrate. This layer has a very low switching field and, due to its thinness [10] a strong in plane-uniaxial anisotropy. $\mathrm{Al}_{2} \mathrm{O}_{3}$ is used as a tunnel barrier and $\mathrm{CoFe}$ as the counter electrode. In this paper, we present the fabrication of the devices, along with the results of magnetoresistance measurements for various field directions and temperatures, and the influence of the anisotropy on the magnetoresistance is explained.

The NiMnSb layer is deposited in a multi-chamber molecular beam epitaxy (MBE) system. An InP substrate with a lattice constant of $5.869 \AA$ close to that of NiMnSb $(5.903 \AA)$ allows for the growth of tens of nm of fully strained NiMnSb[10]. In a first step, a $300 \mathrm{~nm}$ thick highly n-doped (In,Ga)As buffer is deposited on the InP. The sample is subsequently transferred into the NiMnSb growth chamber, where the NiMnSb layer is deposited at a substrate temperature of 300 C. (Detailed growth conditions can be found in ref. [10].) Subsequently, the sample is transferred into a UHV sputtering chamber where a $0.9 \mathrm{~nm}$ thick Al layer is deposited by magnetron sputtering. In a separate chamber, this layer is then oxidized at room temperature in pure oxygen with a pressure of $10^{4} \mathrm{~Pa}$. A second layer of $\mathrm{Al}$ is deposited and again oxidized. With this process, a tunnel barrier with a resistance of approx. $150 \mathrm{k} \Omega \mathrm{cm}$ is obtained. Finally a $10 \mathrm{~nm}$ layer of CoFe is deposited on the AlOx and the sample is covered by a $2 \mathrm{~nm}$ thick Pt layer in order to prevent oxidation.

Mesa structures are fabricated by optical lithography and Argon ion-beam etching down to the highly conductive (In,Ga)As layer. The mesas are passivated by PECVD deposition of a layer of $\mathrm{Si}_{3} N_{4}$. Windows are opened on the top of the Mesa (top contact) and on the (In, Ga)As close to the Mesa (bottom contact). Using optical lithography, deposition of $\mathrm{Ti} / \mathrm{Au}$, and lift off, two large pads are created, which contact top and bottom of the structure through the windows in the $S i_{3} N_{4}$ and allow for bonding far away from the mesa without damaging the TMR contact.

The structures are characterized using a DC transport measurement setup, an electromagnet for room temperature measurements and a superconducting magnet in a ${ }^{4} \mathrm{He}$ bath cryostate for low temperatures. A Quantum Design MPMS SQUID magnetometer is used to determine the switching properties of the magnetic layers at both room temperature and $5 \mathrm{~K}$.

The SQUID measurements are done on samples about $3 \times 3 \mathrm{~mm}$ in size. The magnetic 


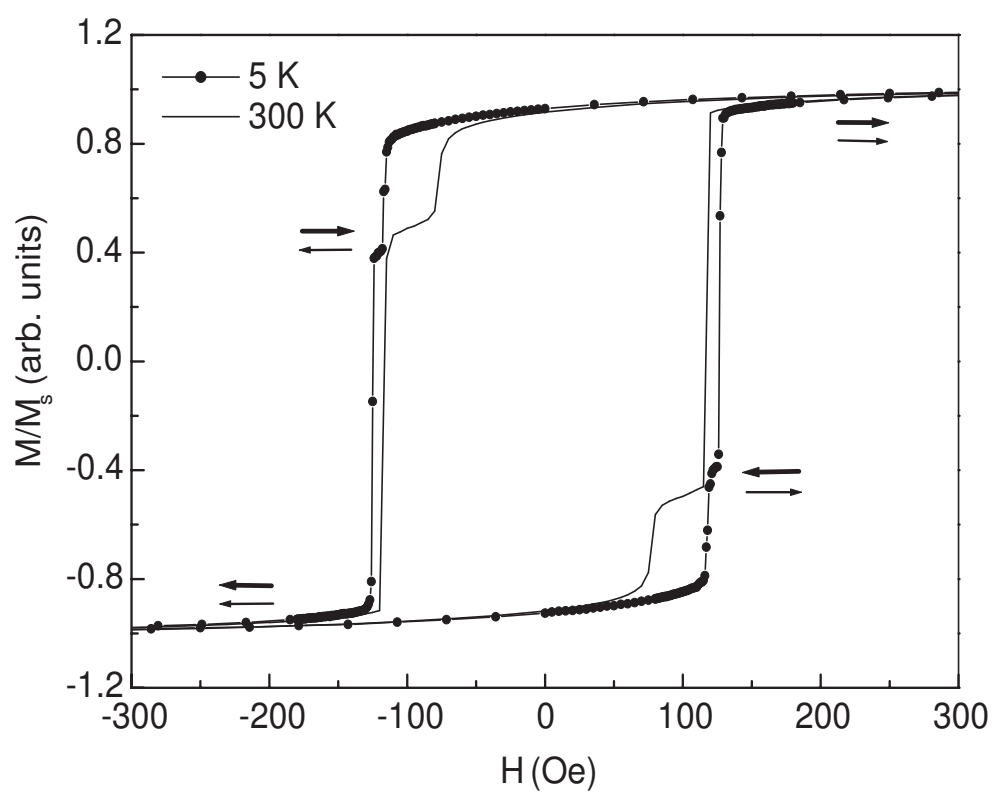

FIG. 1: SQUID magnetization measurements of the TMR stack at 4.2 and $300 \mathrm{~K}$. The signal shows clear separate switching of the two layers.

field is applied in plane and along the easy axis of the NiMnSb layer [110]. The sample shows a clear separate switching of the two magnetic layers both at RT and at $5 \mathrm{~K}$. The CoFe layer shows a coercive field of 115 Oe at room temperature and 126 Oe at $5 \mathrm{~K}$ the coercive field, consistent with expectations. For the NiMnSb layer the coercive field is lower than for the CoFe, however, it is much higher than has been previously observed for single NiMnSb layers. This suggests that some ferromagnetic coupling is present between the two layers. This coupling, which evidently increases at low temperatures, combined with the large magnetic moment of the CoFe layer is responsible for the high coercive field.

\section{RESULTS AND DISCUSSIONS}

$\mathrm{I} / \mathrm{V}$ measurements on the samples at room temperature and at $4.2 \mathrm{~K}$ indicate the presence of a tunnel barrier. The shape of the dI/dV curve can be fitted to Simmon's formula 15] yielding a barrier height of $1.65 \mathrm{eV}$ and a barrier thickness of $1.9 \mathrm{~nm}$ which is in good agreement with the thickness of the deposited $\mathrm{Al}$.

Fig. 2] shows magnetoresistance measurements done with the magnetic field applied along the easy axis of the NiMnSb layer. At room temperature as well as at $4.2 \mathrm{~K}$ the magne- 


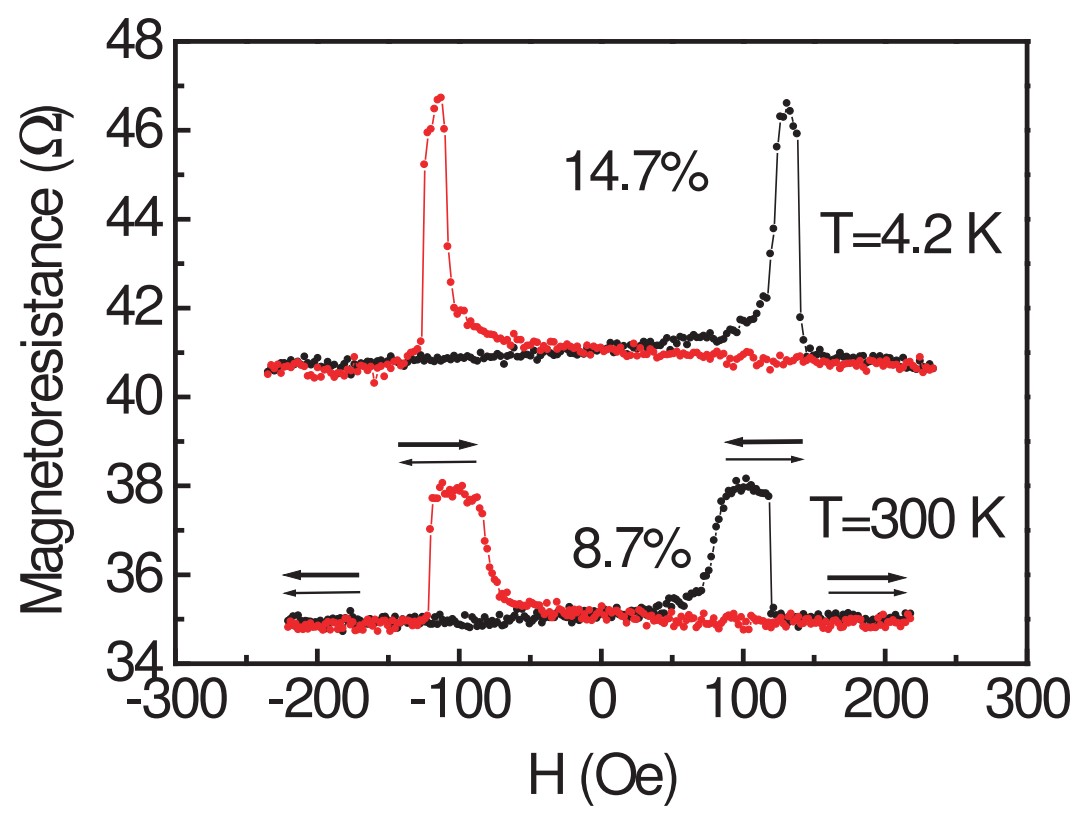

FIG. 2: Magnetoresistance measurements with $\mathbf{H}$ along the easy-axis of the NiMnSb layer at 4.2 and $300 \mathrm{~K}$. The arrows show the magnetization direction for the NiMnSb (thin arrow) and CoFe layers (thick arrow).

toresistance traces have the typical shape expected from a TMR device with independent switching of the two magnetic layers. Only from the high switching field of the NiMnSb and the narrowing of the high resistance region at low temperatures can a ferromagnetic coupling be inferred. The TMR ratio $\Delta R / R$ is $8 \%$ at room temperature and $14 \%$ at 4.2 K. When the magnetic field $\boldsymbol{H}$ is applied along the in-plane hard axis of the NiMnSb as in fig. 3. the shape of the curve is changed. Starting from negative saturation we see a linear increase of the resistance which persists until $\boldsymbol{H}$ has crossed zero. Then at moderate fields the resistance drops by roughly $2 \%$ in a single step and finally drops linearly to the initial saturation value. The saturation field at $4.2 \mathrm{~K}$ is as high as $200 \mathrm{mT}$.

The shape and magnitude of the MR traces for the easy axis measurements are easily explained by taking into account collinear magnetization for the NiMnSb and the CoFe. At large negative fields we have parallel alignment which changes to antiparallel alignment at small positive $\boldsymbol{H}$ when the NiMnSb layer reverses its magnetization and the high resistance (antiparallel) state is obtained (See arrows in fig 21). Further increase of $\boldsymbol{H}$ leads to the 


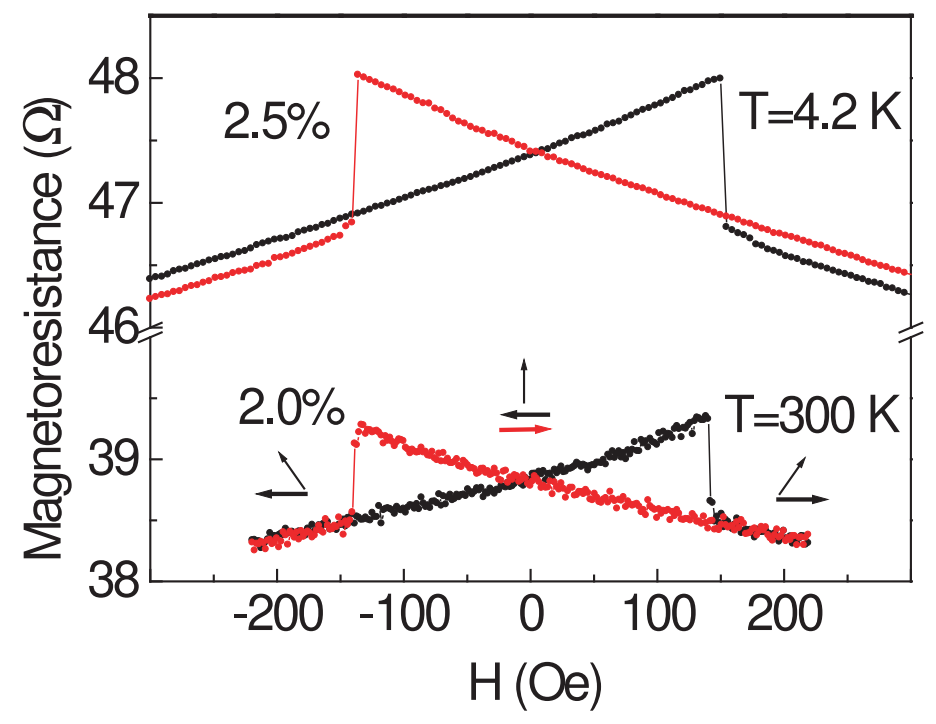

FIG. 3: Magnetoresistance measurements with $\mathbf{H}$ along the hard-axis of the NiMnSb layer at 4.2 and $300 \mathrm{~K}$, respectively. The arrows show the magnetization direction for the NiMnSb (thin arrow) and CoFe layers (thick arrow). The red arrow shows the orientation of the CoFe layer for the downscan

reversal of the CoFe layer and again to a low resistance (parallel) state. For the hard axis measurements we also start with parallel alignment for large negative fields. The crystalline anisotropy of the NiMnSb, however, already starts to dominate at negative fields and the magnetization vector of the NiMnSb layer begins to rotate towards its easy axis which is perpendicular to the magnetic field. This rotation leads to an increase in resistance because the magnetization of the $\mathrm{CoFe}$ and the NiMnSb are no longer collinear. At zero $\boldsymbol{H}$-field the magnetizations are perpendicular to each other. When $\boldsymbol{H}$ is increased beyond zero the magnetization of the NiMnSb slightly rotates towards the antiparallel state and the resistance further increases. At the coercive field of the CoFe layer the resistance exhibits a step down when the magnetization of the CoFe reverses. This step, however, is relatively small because the final state is still an almost perpendicular configuration with only a slightly different projection of the two magnetizations before and after the switching event. Further increase of the field restores the parallel configuration by rotating the magnetization of the $\mathrm{NiMnSb}$ back to the direction of the magnetic field. The fact that nowhere in this scenario an antiparallel configuration appears explains the reduced total MR with respect to the easy 
axis values.

\section{SUMMARY}

TMR devices, which show a TMR of up to $14 \%$ at low temperature have been fabricated based on epitaxial NiMnSb layers. While this value is still below those reported for conventional metallic ferromagnets, further optimization may improve the magnetoresistance ratio. The devices presented, however, have a strong uniaxial anisotropy which has not been observed in other NiMnSb based tunnel junctions and which can be useful for sensor applications, especially, when an angular dependence of the signal is required.

\section{Acknowledgments}

The authors thank V. Hock for processing. The work was supported by the German BMBF (VDI), and the DARPA SpinS program.

[1] J. S. Moodera, L. R. Kinder, T. M. Wong and R. Meservey, Phys. Rev. Lett. 74, 3273 (1995).

[2] T. Miyazaki and N. Tezuka, J. Magn. Magn. Mater. 139, L231 (1995).

[3] J. S. Moodera, J. Nassar and G. Mathon, Annu. Rev. Mater. Sci. 29, 381 (1999).

[4] S. A. Wolf, D. D. Awschalom, R. A. Buhrman, J. M. Daughton, S. von Molnar, M. L. Roukes, A. Y. Chtchelkanova, and D. M. Treger, Science 294, 1488 (2001).

[5] R. Jullière, Phys. Lett. A54, 225 (1975).

[6] M. Viret, M. Drouet, J. Nassar , J. P. Contour , C. Fermon and A. Fert, Europhys. Lett, 39, $545(1997)$

[7] F. Heusler, Verh. Dtsch. Phys. Ges. 5, 219 (1903).

[8] R. A. de groot, F. M. Mueller, P. G. van Engen, and K. H. J. Buschow, Phys. Rev. Lett. 50, 2024 (1983).

[9] W. Van Roy, J. D. Boeck, B. Brijs and G Borghs, Appl. Phys. Lett. 77, 4190 (2000).

[10] P. Bach, A. S. Bader, C. Rüster, C. Gould, C. R. Becker, G. Schmidt, L. W. Molenkamp, W. Weigand, C. Kumpf, E. Umbach, R. Urban, G. Woltersdorf and B. Heinrich, Appl. Phys. Lett., 83, 521 (2003). 
[11] K.E.H.M. Hanssen, P.E. Mijnarends, Phys. Rev. B 34, 5009 (1986)

[12] K.E.H.M. Hanssen, P.E. Mijnarends, L.P.L.M. Rabou, K.H.J. Buschow, Phys. Rev. B 42, $1533(1990)$

[13] C.M. Fang, G.A. de Wijs, R.A. de Groot, Journal Appl. Phys. 91, 8340 (2002)

[14] C. T. Tanaka, J. Nowak and J. S. Moodera, J. Appl. Phys. 81, 5515 (1997), ibid. 86, 6239 (1999).

[15] J. G. Simmons, J. Appl. Phys. 34, 1793(1963). 